\title{
Focal dermal hypoplasia
}

\begin{abstract}
Focal dermal hyperplasia (FDH) or mesoectodermal hyperplasia is an infrequent $\mathrm{X}$-linked dominant patterned inheritable genodermatoses. It compromises skin, bones, eyes, soft tissues and shows different severity degrees. We report the case of a 14-year-old female patient with typical skin lesions of FDH and clinical and histologic findings according to this pathology.
\end{abstract}

Keywords: focal dermal hipoplasia, genodermatoses, limbs skin, cribriform, atrophymacules
Volume I Issue 4 - 2017

\author{
Marcela Alzate Torres,' Gladys Castillo, ${ }^{2}$ \\ Karla Aguilar, ${ }^{3}$ Veronica Posso, ${ }^{4}$ Yadira \\ Aguilar, ${ }^{3}$ Escalante Luis, ${ }^{5}$ Aparicio Rosa' \\ 'Dermatologist, Carlos Andrade Marin Hospital Quito Ecuador, \\ Ecuador \\ ${ }^{2}$ Dermatology, Universidad Central del Ecuador, Ecuador \\ ${ }^{3}$ Resident medical doctor, Carlos Andrade Marin Hospital, \\ Ecuador \\ ${ }^{4}$ Pathologist, Carlos Andrade Marin Hospital, Ecuador \\ ${ }^{5}$ Dermatology, Central University of Ecuador, Ecuador
}

Correspondence: Marcela Alzate Torres, Dermatologist, Carlos Andrade Marin Hospital Quito Ecuador, Quito, Ecuador, Tel 593-984649940,E mail m4rcel4@hotmail.com

Received: November 13, 2017| Published: December 08 2017

\section{Introduction}

Goltz syndrome also known as focal dermal hypoplasia (OMIM\#305600) is a rare dermatoses which was first described by Goltz in the year 1962. It is considered a sporadic genodermatoses (95\% of the patients), although some family-transmitted cases have been reported. This entity compromisesecto and mesoderm-derived tissues, it is predominant in the female sex since it is inherited as an X-linked dominant condition, due to PORCN (locus Xp11.23) gene mutation. As a consequence, the results are lethal for most men. ${ }^{1,2}$ Due to the rarity of the syndrome, the exact prevalence is unknown, just over 300 cases have been reported worldwide. ${ }^{3}$

\section{Case report}

14-year-old female patient, product of a first pregnancy, nonconsanguineous parents, is born at 38 weeks by natural delivery without any complications. There were no interest records in the family. She has a growth retardation and mild mental retardation background. She was addressed to dermatological consultation because she presented widespread skin lesions since birth. In the clinical examination her face symmetry with facial contouring was triangular, hypo pigmented atrophic lesions and erythematosus perioral papules, dental alteration (Figure 1) accompanied by cavities and low implantation prominent ears were found (Figure 2). She had, in her thorax and limbs skin, hypo pigmented, cribriform atrophymacules that alternate with hyper pigmented lesions in a Blaschkoid distribution predominantly in the right hemi-body (Figure 3). Some of her finger and toe nails were hyperplasia (Figure 4). She has skin-color verruciformis papules in her anogenital region.

A skin biopsy was done and found hyperkeratosis skin, irregular hyperplasia of the epidermal rete ridges accompanied by mild and focal basal hyper pigmentation (Figure 5), papillary dermis presents a little lymphocyte infiltration and several small dilatedvessels accompanied by an increase of fat cells to the dermis. Focal rise of fatty tissue in the narrow (Figure 6). X-rays show decreased bone mineralization in the hands and feet and sclerotic joints in the feet. Her skull CTS, pelvic and thyroidal ultrasound were all normal. The patient has 46xx Blood cerotype, 17 hydroxy progesterone hormones, ACTH, normal AM cortisol. Thyroidal examination alteration, sub-clinic hypothyroidism, under pediatrics endocrinology treatment using levothyroxine.

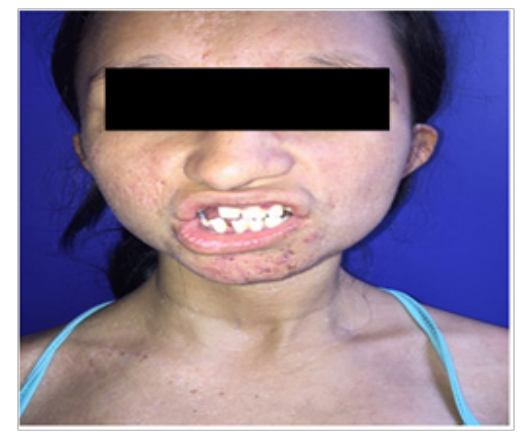

Figure I Alteration of dental morphology.

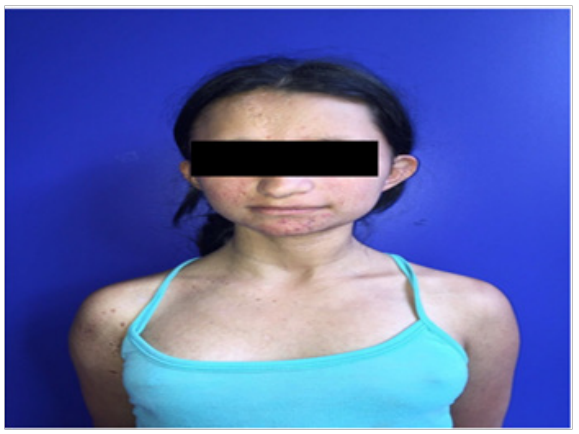

Figure 2 Face asymmetry with triangular facial contouring, hypopigmented atrophic lesions and erythematous perioral papules. 


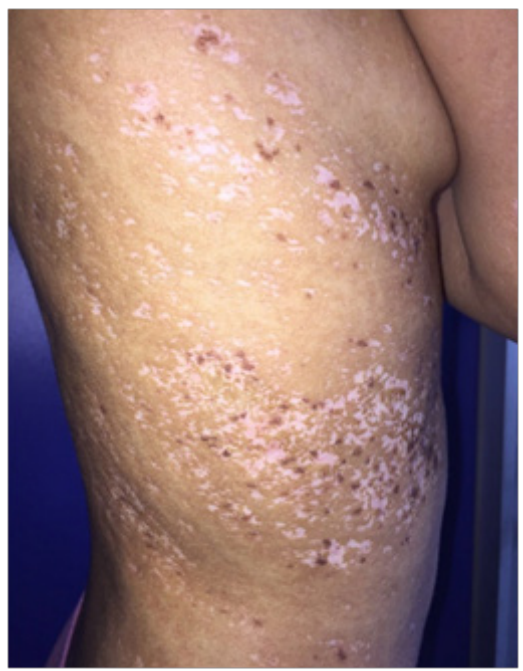

Figure 3 Hypopigmented, cribriform atrophy macules that alternate with hyperpigmented lesions in a Blaschkoid distribution predominantly in the right hemibody.

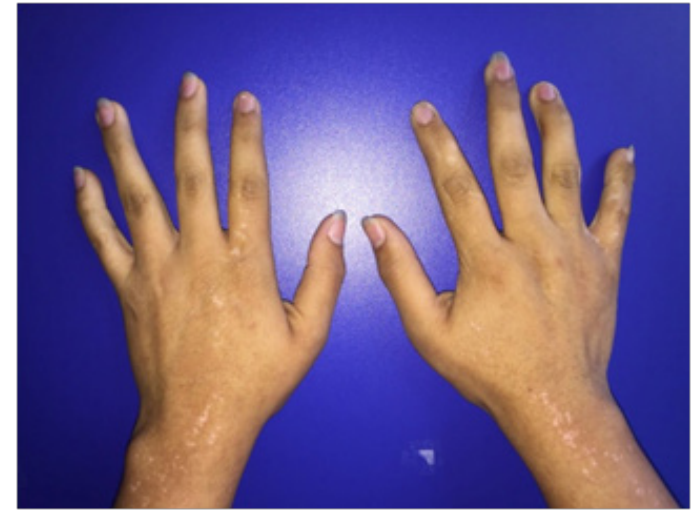

Figure 4 Some hypoplasic nails.

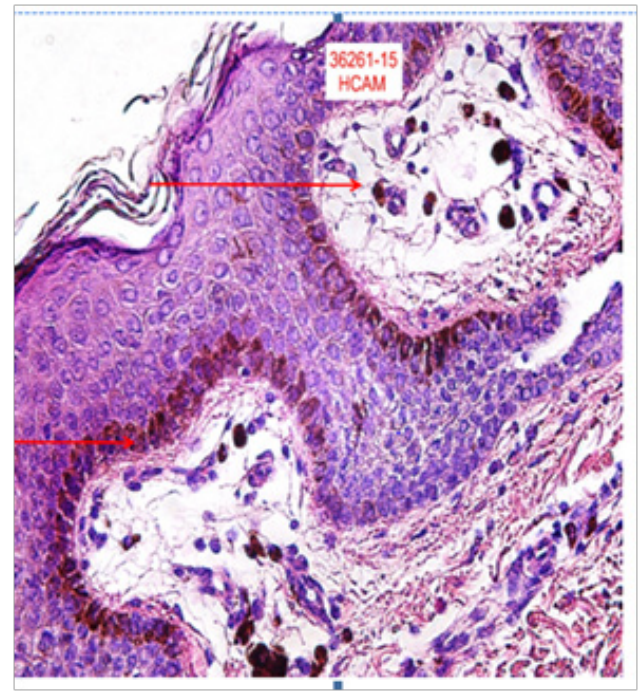

Figure 5 Hyperkeratosis skin, irregular hyperplasia of the epidermal rate ridges accompanied by mild and focal basal hyper pigmentation.

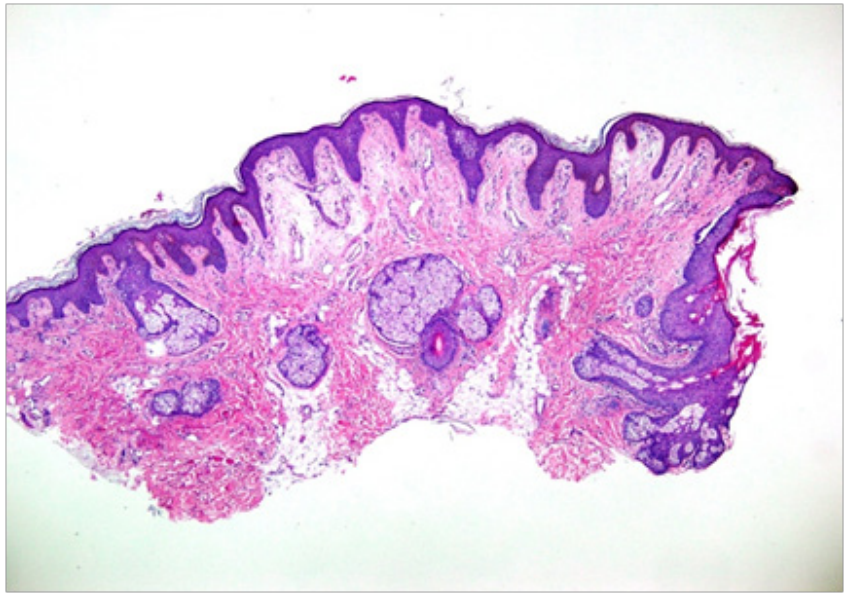

Figure 6 Papillary dermis presents a little lymphocyte infiltration and several dilated small vessels accompanied by an increase of fat cells to the dermis.

\section{Discussion}

Focal dermal hyperplasia is a rare syndrome which multisystemically compromises different impairment degree son skin, eyes and skeleton. It is predominant in the female sex (9:1), it is progressive and could be lethal for men, however only $12 \%$ of male cases have been reported, with regard to genetic mosaicism or de novo gene mutation. It has been noticed that PORCN gene is compromised which codifies the $\mathrm{O}$ acetyltransferase enzyme that is involved in the Want pathway direction. This intercedes in the ecto and mesodermal development, most of the cases the alteration is unilaterally predominant, there are only unilateral alteration cases. ${ }^{4,5}$

Among the manifestations that characterize this entity presents growth retardation, thin Blaschko linear skin lesions, the ones that correspond to the neuronal migration during the embryonic and fetal development producing linear cribriform atrophy accompanied by telangiectasias. Due to the fact that the dermis and other adjacent structures6 are reduced or absent, contribute to subcutaneous cell tissue herniation. Additionally, mucosa fibrovascular papillary lesions are evident in and around the mouth, causing itching and photosensitivity. ${ }^{6,7}$

The fancies features are asymmetrical including triangular facial contouring, microcephaly, sharp chin, ear protrusion, auricle malformation due to poor cartilage development, nasal ala hyperplasia, $30 \%$ alopecia or thin hair, dental hypodontia and microdontia. Also, there is enamel alteration that results in cavities and dental eruption retardation or teeth retention. Among the eye alterations, coloboma, microphthalmia, strabismus, nystagmus, subluxation of the lens of the eye, tear duct cysts, keratoconus, microcornea, cataract, blindness and anophthalmia were all observed. Oligodactyly, bilateral thumb hypoplasia, atrophic concave nail plate or stretch marks and eventually anonychia are evident in hands and teeth.

Among the skeletal alterations it is mentioned, syndactyly with ectrodactyly, finger hyperplasia agenesis, and a radiological pattern in striated osteopathy are observed. Also observed were elongated bones due to chromosomic mosaicism but this finding is not pathognomonic.

Throughout the systemic manifestations are renal agenesis, renal morphology alterations, corpus callosum agenesis and hydrocephalus, 
mental retardation, diaphragmatic hernia and duodenal atresia10. Initially the peculiar and clear clinical background of this syndrome guides us to diagnosis. Nevertheless, nowadays there are specific molecular researches that help us obtain a quick and accurate diagnosis which at the same time allows us to genetically advise properly. In the histological study, the dermis is usually thin due to the lack of connective tissue with an accentuation in the collagen fibers, fat cells are extended to the epidermis mingling with the connective tissue and the epidermis may present acanthosis and papillomatosis. The electron microscopy shows an increase of collagen type III, mainly in the dermis with lack of collagen type IV in the basal membrane4.

The differential diagnosis should considering continentiapigmenti, MIDAS syndrome(microphthalmia, dermal aplasia and sclera cornea), EEC syndrome(ectodermal dysplasia with ectrodactyly, cleft lip and palate), Rothmund-Thoomson syndrome (poikiloderma, and lack of fat hernias common in HDF), epidermolysis bullosa, congenital skin aplasia (characterized by lesions that have lack of local skin on scalp and more oddly on the torso and limbs) superficial lipomatous nevus and Adams-Oliver4 syndrome. The treatment is multi-disciplinary and is directed towards aesthetic and functional alterations each patient uniquely presents. Apart from the opportune genetic advising to the patient and his relatives..$^{8-10}$

\section{Acknowledgements}

Espin Victor MD provided genetic assistance.

\section{Conflict of interest}

This manuscript has not been published and is not under consideration for publication elsewhere. We have no conflicts of interest to disclose.

\section{References}

1. Clements S, Mellerio J, Holden S, et al. PORCN gene mutations and the protean nature of focal dermal hypoplasia. $\mathrm{Br} J$ Dermatol. 2009;160(5):1103-1109.

2. Beatriz A, Saleme C, Juárez F, et al. Hipoplasia dérmica focal. Dermatol Argent. 2011;17(4):306-309.

3. Adeyemi Fowode OA, Mansouri R, Dietrich JE. Gynecologic findings in goltz syndrome: a case series. Am J Med Genet C Semin Med Genet. 2016;172(1):64-66.

4. Acosta J, Motta A, Prieto J. Hipoplasia dérmica focal (Síndrome de Goltz): amplia variabilidad fenotípica. Rev Argent Dermatol. 2009;90(4).

5. Maalouf D, Mégarban H, Chouery E. A novel mutation in the PORCN gene underlying a case of almost unilateral focal dermal hypoplasia. Arch Dermatol. 2012;148(1):85-88.

6. Shimaoka Y, A Hatamochi, Y Hamasaki. Severe focal dermal hypoplasia in a female patient transmitted from a mildly affected mother. $J$ Dermatol. 2009;33:181-183.

7. Sutton V, Van den Ver I. Focal Dermal Hypoplasia: Gene Reviews. Seattle, USA: University of Washington; 2009.

8. Riyas N, Riyas A, Chandran R, et al. Focal Dermal Hypoplasia (Goltz Syndrome). Indian J Dermat Venereol Leprol. 2005;71(4):279-281.

9. Morales M, Garibay A, Álvarez E, et al. Hipoplasia dérmica focal (Síndrome de Goltz). Rev Cent Dermatol Pascua. 2014;23(1):1-6.

10. Lasocki A, Stark Z, Orchard D. A case of mosaic Goltz syndrome (focal dermal hypoplasia) in a male patient. Australasian J Dermatol. 2011;52:48-51. 